\title{
Die fachkonzeptionelle Gestaltung der Kundenbeziehung als Basis für den Prozessentwurf
}

\author{
Bernd Heinrich
}

In saturierten und wettbewerbsintensiven Märkten muss die Zielsetzung verfolgt werden, den einmal gewonnenen Kunden nachhaltiger an das Unternehmen zu binden, um ihm auch zukünftig die eigenen Dienstleistungen möglichst ausschliesslich anbieten zu können. Derartige Erkenntnisse bedeuten einen Übergang zwischen der kurzfristigen Vermarktung einzelner Produkte und Produktbündel hin zu einer längerfristigen Perspektive, die den Kunden bzw. die Beziehung als Wertgegenstand in den Mittelpunkt der Betrachtung stellt. Wird jedoch der Kunde als „kritische Ressource“ des Unternehmens verstanden, müssen aufgrund der Bedeutung dem Prozessentwurf und dem IT-Einsatz zwangsläufig fachkonzeptionelle Überlegungen vorangehen. Der Beitrag greift diesen Problembereich auf und stellt anhand einzelner praktischer Beispiele ein methodisches Vorgehen zur Bearbeitung der Problematik dar.

\section{Einleitung}

Der Sachverhalt, dass bei Finanzdienstleistern ca. 60-80\% der Privatkunden einen negativen Erfolgsbeitrag bzw. Deckungsbeitrag erbringen, wird schon seit einigen Jahren in Theorie und Praxis ausgiebig erörtert [vgl. Bernet 1998]. Rückführbar ist dies sowohl auf die Erlösseite, die in den letzten Jahren zusätzlich durch den verstärkten Wettbewerb und den damit verbundenen Preisdruck gelitten hat (auch bei Kunden mit höherem Potenzial), wie auf den immer noch hohen Kostenfaktor. Insofern ist aus Unternehmenssicht zu fordern, sich auf profitable Kunden zu konzentrieren (Effizienz), indem dauerhaft deren Bedürfnisse gezielter bzw. umfassender befriedigt werden (Effektivität). Mit speziellem Fokus auf die Kundenbeziehungen bedeutet dies insbesondere, die Nachhaltigkeit und Intensität in den Vordergrund zu stellen. Gerade letztgenannter Aspekt bedarf laut der Studie von Daniel einer Lösung, da z.B. der Prozentsatz von Mehrfachbankverbindungen unter Sparkassenkunden bei durchschnittlich 48\%, bei einkommensstarken Kunden sogar bei 71\% liegt [vgl. Daniel 2001]. 
Für die Lösung derartiger Problemstellungen reicht es jedoch nicht aus, ausschliesslich den Einsatz von Informationssystemen zu erörtern. Vielmehr entsteht die Notwendigkeit fachkonzeptionelle Überlegungen als Basis voranzustellen, was sich mitunter auch in der Vielzahl von CRM-Projekten in der Praxis zeigt, die wegen der fehlenden konzeptionellen Basis scheitern respektive nicht zu den gewünschten Ergebnissen führen [Lotz/Schröder 2002; Nelson 2002]. Nachfolgend wird die Ausarbeitung einer fachlichen Konzeption fokussiert und am Anwendungsfall eines Kreditinstitutes verdeutlicht.

\section{Fachkonzeptionelle Festlegungen im Kundenbeziehungsmanagement}

Bevor einzelne Bindungsmassnahmen zu beschliessen und umzusetzen sind, ist in einem ersten Schritt ein konzeptioneller Rahmen zu schaffen. Dieser determiniert die grundsätzlichen und zugleich effektiven Möglichkeiten bzw. Instrumente, um die eigene Klientel und vor allem die wertvollen Kunden zum Erhalt der Beziehung zu veranlassen. Einer derartigen, konzeptionellen Aufgabenstellung wird jedoch in Theorie und Praxis oftmals ein zu geringer Stellenwert eingeräumt, da vorrangig punktuelle und isolierte Einzelmassnahmen (z.B. Einführung eines Bonussystem, wie dem UBS Key Club) erörtert werden. Insofern wurde im geschilderten Anwendungsfall zuerst geprüft, inwieweit die diskutierten Instrumente von der Klientel des Kreditinstituts überhaupt akzeptiert werden, d.h. ihr Bindungspotential letztlich auch entfalten können.

\subsection{Die Ermittlung der Kundenmotive für den Aufbau und Erhalt einer Beziehung}

Legt man den Grundsatz der Kundenorientierung zugrunde, so sind die Beziehungsmotive des Kundenstamms eines Unternehmens bestimmend für die Definition eines effektiven Instruments. Basierend auf den Ergebnissen einer allgemeinen, explorativen Studie, in welcher zwölf unterschiedliche Kategorien von Beziehungsmotiven identifiziert wurden [vgl. Heinrich 2002b], liessen sich mittels einer Befragung die für den unternehmensspezifischen Anwendungsfall dominanten Motive ermitteln. Hierbei war es notwendig durch den Kunden eine Priorisierung vornehmen zu lassen, d.h. es wurde bspw. gefragt, welche Faktoren (z.B. Bequemlichkeit des Kontakts, monetäre Anreize, besonders sympathisches Bankpersonal, Image des Kreditinstituts etc.) für die Weiterführung der Beziehung respektive für einen Wiederkauf massgeblich ausschlaggebend sind. Interessanterweise ergab sich in diesem Zusammenhang keine Gleichverteilung über alle "möglichen" Beziehungsmotive. Vielmehr fand im Gegensatz zu anderen Untersuchungen eine deutliche (unternehmenscharakteristische) 
Schwerpunktbildung statt, welche die sicherheits- und produktbezogenen Motive besonders betonte. Dies bedeutet, dass ein erheblicher Teil der Kunden bspw. die hohe Erfüllungsqualität der Bank wie auch die empfundene Sicherheit vor opportunistischem Verhalten schätzt und sie zugleich als Basis für die zukünftige Beziehung sieht.

Darüber hinaus kristallisierten sich in Abhängigkeit von der Beziehungsphase (z.B. Kennenlernen, Start, Penetration \& Reife sowie Krise \& Distanzierung [vgl. hierzu auch Diller 1995]) teilweise deutliche Unterschiede in den genannten Motiven heraus. So wurden insbesondere in den Aufbauphasen der Kundenbeziehungen oftmals noch monetärbezogene Gründe angeführt, die jedoch mit zunehmender Dauer an Priorität stark abnahmen. Im Gegensatz dazu wurden kollektivbezogene Motive, welche die soziale Ebene des Kontakts ("sich gut betreut fühlen") repräsentieren, naturgemäss in späteren Beziehungsphasen stärker gewichtet. Zugleich waren sie auch wichtigster Weiterempfehlungsgrund.

Die Abbildung 1 zeigt idealtypisch die Bedeutung (ein grösser Balken entspricht einer höheren Relevanz) verschiedener Beziehungsmotive im zeitlichen Verlauf für den Fall des fokussierten Unternehmens. Dabei spielen, wie oben schon ausgeführt, in den frühen Phasen hauptsächlich produkt- und monetärbezogene Motive (hohe Erfüllungsqualität, punktuelle Preisnachlässe etc.) eine zentrale Rolle. Demgegenüber geben Personen, die schon länger Kunden des Unternehmens sind (Hauptgeschäftsverbindung), neben den produktinsbesondere auch sicherheits- (Kunde befürchtet kein opportunistisches Verhalten der Bank) und kollektivbezogene Gründe als bedeutsam an.

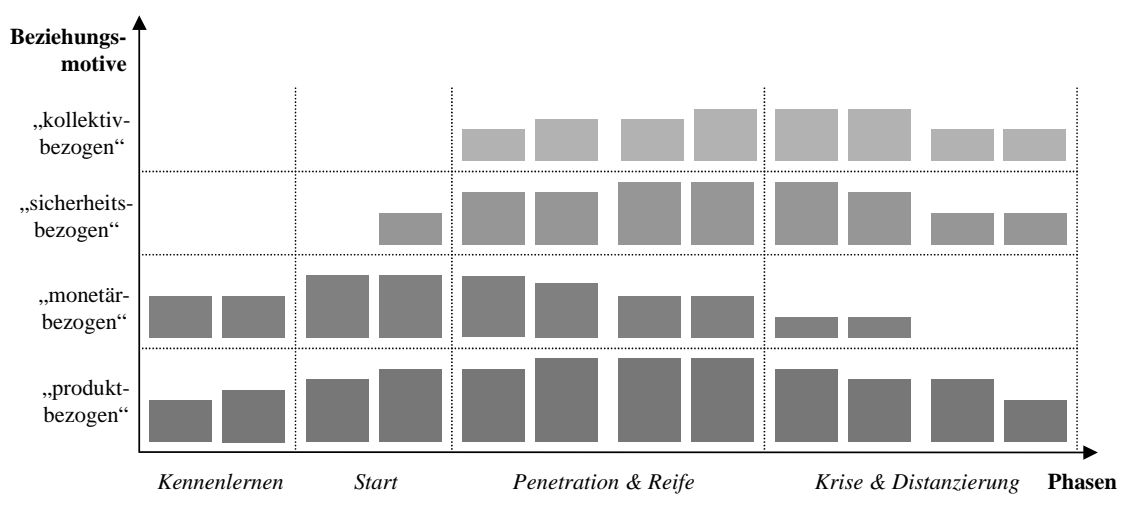

Abbildung 1: Beispiel für die Bedeutung von Kundenmotiven im Beziehungsverlauf

Auf Grundlage derartiger Erhebungen von Beziehungsmotiven liessen sich wiederum verschiedene Kundentypen innerhalb der Klientel identifizieren, welche proaktiv für die nachfolgend dargestellte Definition von Beziehungsträgern und -instrumenten genutzt werden konnten. 
Ein Aspekt, der im weiteren zwar nicht vertieft behandelt wird, jedoch dennoch sehr interessant ist, war die höhere Bewertung von Kunden mit sicherheits- und kollektivbezogenen Motiven. Diese Kundengruppe zeichnet sich dadurch aus, dass sie im Durchschnitt eine intensivere (weniger Mehrgeschäftsverbindungen sowie eine höhere Produktnachfrage beim Kreditinstitut) und längere Beziehung aufrechterhält. Unter normativen Gesichtspunkten sind somit derartige Kundenbeziehungen mit Nachdruck aufzubauen. Dies kann u.a. auch erfolgen, indem bei vorhandenen Kundenbeziehungen versucht wird, sicherheits- und kollektivbezogene Motive proaktiv hervorzurufen. Ein systematisches Vorgehen ist diesbezüglich jedoch noch zu konzipiert und zu validieren.

\subsection{Die Definition der Beziehungsinstrumente zur Bindung des Kunden}

Basierend auf obiger Analyse stellte sich die Frage, welche Träger der Beziehung zwischen den Geschäftspartnern existieren müssen (z.B. ein immaterieller Wert wie Vertrauen), damit einerseits den Kundenmotiven entsprochen werden kann und die zum anderen als Ansatzpunkt für die Definition der unternehmenseigenen Beziehungsinstrumente dienen können. Zur generellen Identifikation der Beziehungsträger wurden dabei ökonomische und verhaltenswissenschaftliche Theorien berücksichtigt [vgl. Heinrich 2002b].

Beispielsweise ergibt sich bei den sicherheitsbezogenen Motiven die Alternative einer Vertrauensbildung beim Kunden, d.h. dieser wird vom integeren Verhalten der Bank (sukzessive) überzeugt. Konträr dazu können dem Kunden jedoch auch Anreiz-, Straf- oder Kontrollmechanismen (z.B. Garantien) zur Verfügung gestellt werden, die im Sinne einer vertraglichen Regelung ein opportunistisches Verhalten der Bank (aus Kundensicht) verhindern sollen. In Abhängigkeit von der Kundenakzeptanz und vor allem auch vom Marktauftritt der Bank kann hierbei eine Selektion bzw. ein kombinierter Einsatz erfolgen. Wirbt die Bank bspw. damit, den Kunden nicht nur umfassend zu beraten sondern vielmehr zu betreuen, so erscheint ein Vertrauensaufbau das weitaus probatere Mittel zu sein.

Stehen derartige, je analysiertem Beziehungsmotiv zu treffenden Entscheidungen fest, sind im nächsten Schritt diejenigen Instrumente zu ermitteln, mittels derer die einzelnen Beziehungsträger (z.B. Vertrauen) konstituiert werden können. Hierbei kann grundsätzlich zwischen einem Personen-, Unternehmens- oder Sachbezug des Instruments unterschieden werden [vgl. Dittrich 2000], d.h. ob bspw. das Kundenvertrauen durch die Eigenschaften des Mitarbeiters oder des Unternehmens (z.B. Reputation) erzeugt wird. Auch hier kann eine Auswahl sowohl unter Berücksichtigung der Kundenakzeptanz wie auch des Marktauftritts (z.B. entfällt für eine Direktbank der Personenbezug meist gänzlich) erfolgen.

Die Abbildung 2 fasst die konzeptionellen Festlegungen nochmals am Beispiel der sicherheitsbezogenen Motive zusammen: 


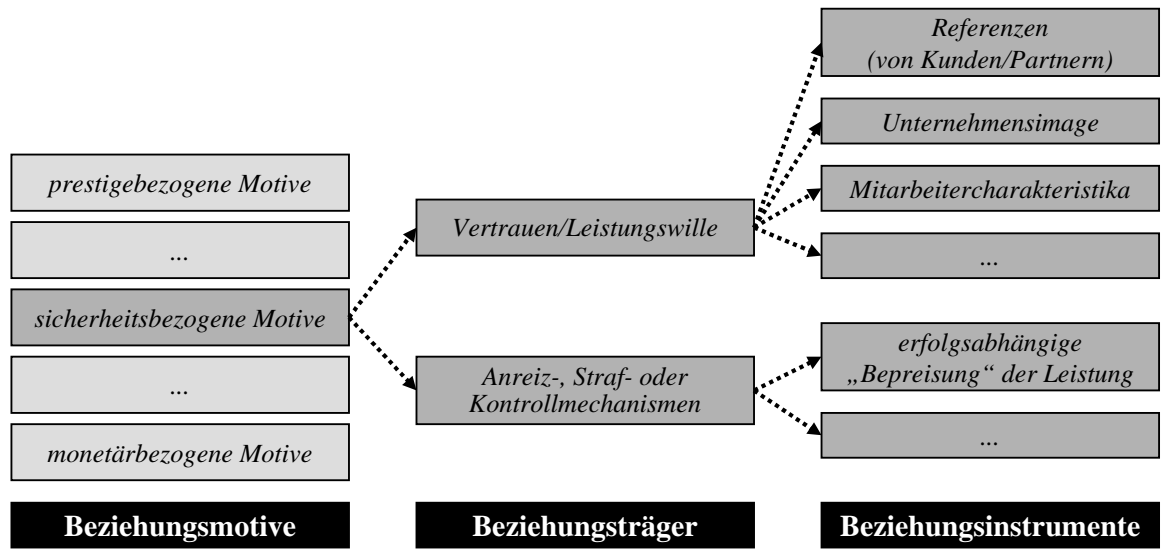

Abbildung 2: Ableitung der Beziehungsinstrumente anhand von Kundenmotiven

\section{Die Umsetzung der Beziehungsinstrumente im ganzheitlichen Vertriebswegemix}

Für die Realisation der bisher noch abstrakt definierten Beziehungsinstrumente, sind diese in die Vertriebsinteraktion mit dem Kunden konkret zu berücksichtigen. Für eine systematische Durchführung, müssen die Vertriebsaktivitäten, welche ihrerseits auf einem unmittelbaren Kundenbedürfnis basieren, transparent und möglichst ganzheitlich dargestellt werden. Ganzheitlichkeit bezieht sich dabei auf die Kundensicht, d.h. es sollen alle wesentlichen Schritte für die Bewältigung eines Kundenbedürfnisses durchgängig veranschaulicht werden. Als Ausgangspunkt für die Identifikation der Bedürfnisse sollen in diesem Zusammenhang Lebensereignisse oder spezielle -situationen des Kunden dienen [vgl. Winter 2002], wie bspw. der Erwerb von Wohneigentum oder das Gründen eines eigenen Kleinbetriebs.

\subsection{Die ganzheitliche Modellierung einer Geschäftstransaktion}

Sollen die primären Aktivitäten des Kunden zur Bewältigung eines seiner Bedürfnisse sowie deren Reihenfolge modelliert werden, so stellt der Kundenprozess ein probates Mittel dar [Österle et. al. 2000; Rapp 2000]. Dieser integriert die verschiedenen Phasen der Bedürfnisentstehung, -formulierung, -evaluierung, -bewältigung und -bestätigung, wobei jedoch nicht auf einen isolierten Buying-Cycle, wie z.B. für die Inanspruchnahme eines Kredits, abgezielt wird. Vielmehr soll das Gesamtproblem des Kunden bei Eintritt eines 
Lebensereignisses oder einer Lebenssituation zugrunde gelegt werden, auch um mit der hierdurch angestrebten Leistungsintegration einen Mehrwert für den Kunden zu schaffen [vgl. Heinrich 2002a].

Am Beispiel der Lebenssituation "Gründen eines eigenen Kleinbetriebs" lässt sich die Modellierung eines Kundenprozess veranschaulichen. Wie die Abbildung 3 mit dem auf der linken Seite dargestellten Kundenprozess (verkürzte Version) zeigt, sind mehrere Stationen mit unterschiedlichen Geschäftspartnern zu durchlaufen, damit das Gesamtproblem umfassend bewerkstelligt werden kann. Aufgrund der Komplexität bieten sich mehrere Ansatzpunkte für die Unterstützung seitens eines sogenannten Service Integrators an. Dieser gleicht einem Intermediär, der Produkte und Dienstleistungen vermittelt respektive zu einer individuellen Lösung für den Kunden bündelt [vgl. Leist/Winter 2000]. Ein Kreditinstitut, welches die Rolle eines Service Integrators einnimmt, müsste dementsprechend ganzheitliche Lösungen, in Zusammenarbeit mit „branchenfremden“ Partnerunternehmen, anbieten.

Interessant erscheint dabei bspw. der Umstand, dass durch die Betreuung „aus einer Hand“, mehrmaliges und mühsames Erklären von bestimmten Sachverhalten (z.B. der Geschäftsidee) für den Kunden entfällt. Ebenso wertvoll ist das Führen und Lenken des Prozesses durch den Service Integrator, da in den allermeisten Fällen der Kunde nicht alle zu erledigenden Details kennt bzw. er nicht die Zeit zu deren Durchführung besitzt. Darüber hinaus ist hierbei auch die Technikaffinität des Kunden zu untersuchen [vgl. Bartmann 1997; Spiegel 2000], um z. B. festzulegen welche Schritte des obigen Prozesses ohne Benutzung elektronischer Medien zu erfolgen haben. Ebenso könnten die zeitlichen Präferenzen des Kunden aufgrund seiner Berufstätigkeit ausserhalb der üblichen Öffnungszeiten von Dienstleistern (im Finanzsektor) liegen, was es entsprechend zu berücksichtigen gilt. 


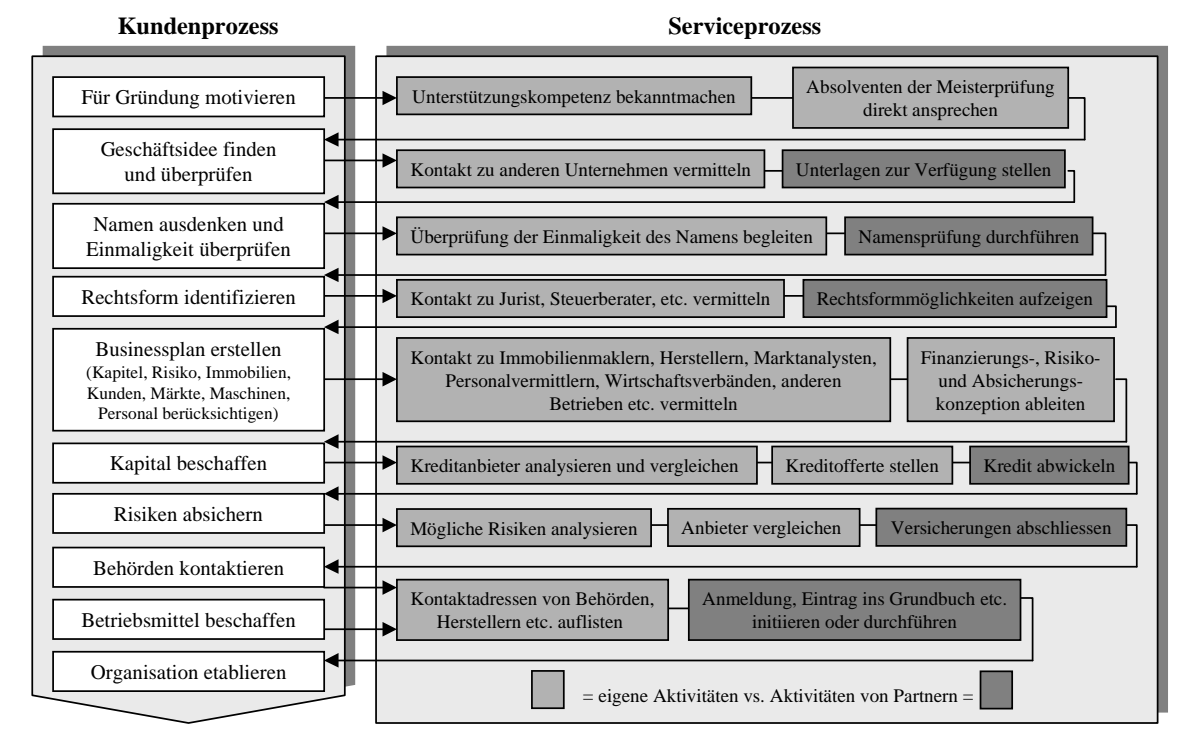

Abbildung 3: Kunden- und Serviceprozess zur

Lebenssituation "Gründen eines eigenen Betriebs"

Zugleich zeigt die Abbildung 7 rechts den Serviceprozess in komprimierter Form, wie er für einen convenienceorientierten Service Integrator sinnvoll erscheint. Durch die Orientierung am Kundenprozess wird eine ganzheitliche und durchgängige Unterstützung gewährleistet. Für jede Aktivität kann dabei entschieden werden, ob man diese selbst durchführt (in der Abbildung hell gekennzeichnet) und welche Rolle man für den Fall einer Übernahme durch Kooperationspartner einnehmen möchte. Zudem lassen sich sehr einfach die einzelnen Leistungen und Produkte, aber auch Werbestimuli (z.B. Bekanntmachung der eigenen Kompetenz in Fachzeitschriften) zuordnen und placieren. Generell zu beachten ist dabei die Kompensationsbereitschaft der Klientel. Wird diese als durchschnittlich eingeschätzt, so ist eine gewisse Standardisierung und straffe Organisation erforderlich. Dem könnte bspw. für die Aktivität „Business Plan erstellen“ Rechnung getragen werden, indem bei der Erstellung und Prüfung der Pläne der einzelnen Unternehmensgründer, die hierzu nötigen Workshops zeitlich und räumlich koordiniert sind. Dies ermöglicht als Mehrwert zugleich einen Erfahrungs- und Wissensaustausch zwischen den Kunden.

Zusammenfassend lässt sich als wesentlicher Vorteil der Modellierung von Kunden- und Serviceprozess insbesondere die Möglichkeit einer systematischen Planung der Austauschbeziehungen zwischen Kunde und Unternehmen nennen. Zudem bietet die umfassende Bearbeitung eines Bedürfnisses eine grosse Chance zur Intensivierung der Zusammenarbeit, da dem Kunden bspw. die Mühe 
abgenommen wird, sein (für ihn eventuell neues) Bedürfnis zu lösen, d.h. herauszufinden, welche einzelnen Leistungen er benötigt, welche die besten Leistungen für ihn sind etc. Es gelingt somit einerseits mittels des Kundenprozesses ein Verständnis für die Probleme, Präferenzen und Abneigungen sowie die Lebens- und Denkwelt des Kunden zu generieren und zu operationalisieren. Andererseits bietet der Serviceprozess die Möglichkeit eines konzeptionellen Rasters für die Partnersuche.

\subsection{Die Ausgestaltung der Beziehungsinstrumente innerhalb einer Geschäftstransaktion}

Neben seiner Zweckmässigkeit bei der Bewältigung eines Kundenbedürfnisses soll der Serviceprozess insbesondere auch zur Berücksichtigung der Beziehungsinstrumente dienen, da dieser die faktische Interaktion mit dem Kunden beschreibt. Demzufolge sind die einzelnen Leistungsaktivitäten im Prozess dahingehend zu untersuchen, inwieweit sie Ansatzpunkte für den Einsatz der Instrumente aufzeigen, d.h. bei der Aktivitätsdurchführung eingeplant werden können. Dies entspricht gleichzeitig einer Konkretisierung der Beziehungsinstrumente, die ihrerseits nunmehr in einzelne Massnahmen und Aktionen aufzugliedern sind.

Im obigen Anwendungsfall wurden auszugsweise einige Beziehungsinstrumente genannt, mittels derer den sicherheitsbezogenen Motiven entsprochen werden soll. Beispielhaft lassen sich hier Kunden- und Partnerreferenzen zur Vertrauensbildung anführen, die sich innerhalb des Serviceprozesses in Abbildung 3 insbesondere in den Aktivitäten "Unterstützungskompetenz bekanntmachen", "Kontakt zu anderen Unternehmen vermitteln (um die Tragfähigkeit der neuen Geschäftsidee zu beurteilen)" und "Kontakt zu Herstellern, Wirtschaftsverbänden etc. vermitteln (bei der Erstellung des Businessplans)" einplanen lassen. Dies beruht darauf, dass hier der Kunde vom Leistungswillen und der -fähigkeit des Unternehmens aufgrund von bisherigen Erfahrungswerten überzeugt werden soll. Operativ kann dies innerhalb der oben schon angesprochenen Workshops mit den zukünftigen Firmengründern umgesetzt werden. Gleichermassen kann hierbei auch von der Einbindung und der Zusammenarbeit mit namhaften Geschäftspartnern des Kreditinstituts (z.B. Geschäftspartner der Versicherungsbranche) profitiert werden, wenn diese über eine entsprechende Reputation im Bereich der Unternehmensgründung besitzen. In analoger Weise sind die anderen Instrumente zum Aufbau und zur Generierung von Vertrauen im Serviceprozess zu berücksichtigen, d.h. es bspw. zu prüfen, in welchen Schritten und bei welchen Kunden gezielt erfahrene und routinierte Mitarbeiter eingesetzt werden müssen.

Im Gegensatz zu den Massnahmen der Vertrauensbildung kann den sicherheitsbezogenen Kundenmotiven bspw. auch durch eine erfolgsabhängige 
Bepreisung oder durch Garantien entsprochen werden (siehe Abbildung 2). Ziel derartiger Mechanismen ist es, die Unsicherheit des Kunden über das Verhalten des Unternehmens $\mathrm{zu}$ reduzieren oder $\mathrm{zu}$ zerstreuen, indem eine Interessensangleichung vorgenommen. Dies kann erfolgen, indem der Preis bzw. die Gebühr der Geschäftstransaktion in vollem Umfang vom Kunden nur für den Erfolgsfall zu leisten ist, d.h. wenn das Ergebnis der Zusammenarbeit einem zuvor vereinbarten Niveau entspricht. Insofern wird der Service Integrator bzw. die Bank während der Dauer des Geschäfts die Anstrengungen aufrechterhalten, um das definierte Vertragsergebnis (z.B. Vermittlung geeigneter Verträge und Partner für die Kapitalbeschaffung oder Risikoabsicherung) zu erreichen. Im Vermögensgeschäft der Banken werden derartige Mechanismen bereits punktuell bspw. im Sinne einer garantierten Mindestrendite bei risikobehafteten Anlageformen (z.B. Anlagefond mit Aktienanteil) eingesetzt. Die Vorteile im Kontakt mit dem Kunden sind dabei offensichtlich, da Letztgenannter ohne den direkten Vertrauenseffekt einer langjährigen Geschäftsbeziehung, seine Befürchtungen hinsichtlich eines opportunistischen Verhaltens der Bank (z.B. beim Management des Fonds) verringert sieht. Entsprechend könnten Kontroll-, Anreiz- und Strafmechanismen auch im obigen Serviceprozess eine Berücksichtigung finden.

Neben der Integration einzelner Instrumente erhöht, so die praktischen Erfahrungen, insbesondere die Durchgängigkeit in der Betreuung des Kundenprozesses die Dauer und Intensität der Beziehung. Dies ist darauf zurückzuführen, dass infolge des ganzheitlichen Leistungsangebots, ein Kunde für sein Problem nicht mehr eigenhändig geeignete Lösungsmöglichkeiten (meist in Form von einzelnen Dienstleistungen oder Produkten) langwierig suchen, bewerten und mühsam integrieren muss. Auch aus Sicht der Kreditinstitute hat der Zusammenschluss mit Partnern in einem Geschäftsnetzwerk wesentliche Vorteile, da hierdurch der Entwicklung der originären Bankdienstleistung zur "commodity" (aufgrund der Angebotshomogenität) entgegengewirkt werden kann. Durch die ganzheitliche Unterstützung des Kunden im Sinne seines Grund- oder Gesamtbedürfnisses gilt es insofern, wieder mehr Differenzierungspotential für eine längerfristige und intensive Beziehung zu generieren.

\section{Fazit}

Will man seine Kundenbeziehungen nachhaltig und intensiv gestalten, so ist eine Kenntnis der Motive der eigenen Klientel für den Aufbau und Erhalt der Geschäftsverbindung unumgänglich. Nur auf diese Weise ist ein effektiver Einsatz von Instrumente und Massnahmen zur Bindung des Kunden möglich. Die Untersuchung und Differenzierung der Beziehungsmotive zeigt jedoch noch einen weitergehenden Aktionsspielraum auf. Fasst man nämlich die Existenz und 
Priorität der Kundenmotive nicht als feststehend auf, so können diejenigen Motive, die empirisch zu einer besonders nachhaltigen und intensiven Zusammenarbeit führen, proaktiv gefördert werden. Im geschilderten Praxisfall wurde darum in erster Linie versucht, die sicherheits- und kollektivbezogenen Motive nachdrücklicher in den Mittelpunkt der Anstrengungen zu stellen und diese gewissermassen beim Kunden zu initiieren. Unbenommen der schwierigen Umsetzung einer derartigen, normativen Richtlinie, sollen somit wertvolle Kunden stärker an das Unternehmen gebunden werden.

Daneben wurde im Beitrag auch die Operationalisierung und Integration einzelner Beziehungsinstrumente in einer Geschäftstransaktion gezeigt. Ausgehend von der Modellierung des Kundenprozesses, der die Schritte zur Bedürfnisbewältigung repräsentiert, wird ein Serviceprozess entwickelt. Dieser integriert die Leistungen verschiedener Unternehmen in einem Geschäftsnetzwerk zu einem ganzheitlichen Lösungsangebot. Zugleich können auf dieser Basis übergreifende Bindungsmassnahmen konzipiert und berücksichtigt werden, die sowohl preisliche oder produktbezogene Verbundvorteile wie auch immaterielle Faktoren (z.B. Vertrauen) umschliessen können.

Die Einplanung der Instrumente in die Geschäftstransaktionen ist jedoch nur ein erster wichtiger, konzeptioneller Schritt für faktische Umsetzungen im Kundenbeziehungsmanagement. Zukünftige Aufgabenfelder im Kompetenzzentrum müssen explizite Beziehungsprozesse, wie bspw. die Kundenrückgewinnung oder -reaktivierung ebenso einbeziehen, da deren Erfolgsaussichten wesentlich von den aufgebauten Beziehungswerten (z.B. Vertrauen) bestimmt werden [vgl. Homburg/Schäfer 1999; Stauss 2000].

\section{Literaturverzeichnis}

Bartmann, D. (1997): Die drei Dimensionen der ibi-Finanztypologie: Unterlagen des Instituts für Bankinformatik der Universität Regensburg, Regensburg.

Bernet, B. (1998): Konzeptionelle Grundlagen des modernen Relationship Banking, in: Bernet, B.; Held, P. P. (Hrsg.): Relationship Banking: Kundenbeziehungen profitabler gestalten, Wiesbaden, S. 3-36.

Daniel, J. (2001): Ertragssteigerung durch Kundenzufriedenheit und Kundenbindung. Möglichkeit und Grenzen am Beispiel eines Kreditinstituts, Stuttgart.

Diller, H. (1995): Kundenbindung als Zielvorgabe im Beziehungs-Marketing, Arbeitspapier Nr. 40, Institut für Marketing, Universität Nürnberg-Erlangen, Nürnberg.

Dittrich, S. (2000): Kundenbindung als Kernaufgabe im Marketing, Dissertation der Universität St. Gallen, Schesslitz. 
Lotz, W./Schröder, T. (2002): CRM-Systeme: Trends, Bewertungen und Auswahl, Computerwoche-Online (www.computerwoche.de), 22.01.2002.

Heinrich, B. (2002a): Die konzeptionelle Gestaltung des Multichannel-Vertriebs anhand von Kundenbedürfnissen, in: Leist, S., Winter, R. (Hrsg.): Retailbanking im Informationszeitalter - Integrierte Gestaltung der Geschäfts-, Prozess- und Applikationsebene, Berlin, S. 73-91.

Heinrich, B. (2002b): Methode zur wertorientierten Analyse und Gestaltung der Kundeninteraktion - Zur Rolle des Service Integrators im Privatkundengeschäft von Kreditinstituten, Dissertation der Universität St. Gallen, Erscheint im Logos-Verlag.

Homburg, C./Schäfer, H. (1999): Customer Recovery, Institut IMU, Universität Mannheim.

Leist, S./Winter, R. (2000): Finanzdienstleistungen im Informationszeitalter - Vision, Referenzmodell und Transformation, in: Belz, C.; Bieger, T. (Hrsg.): Dienstleistungskompetenz und innovative Geschäftsmodelle, St. Gallen, S. 150-166.

Nelson, S. (2002): What's Happening to CRM in 2002, Gartner Group Research, January 2002.

Österle, H./Fleisch, E./Alt, R. (2000): Business Networking: Shaping Enterprise Relationships on the Internet, Springer, Berlin.

Rapp, R. (2000): Customer Relationship Management: das neue Konzept zur Revolutionierung der Kundenbeziehungen, Frankfurt am Main.

Spiegel (2000): Soll und Haben 5: Dokumentation, Spiegel-Verlag, Hamburg.

Stauss, B. (2000): Rückgewinnungsmanagement: Verlorene Kunden als Zielgruppe, in: Bruhn, M.; Stauss, B. (Hrsg.): Dienstleistungsmanagement Jahrbuch 2000: Kundenbeziehungen im Dienstleistungsbereich, Wiesbaden, S. 449-471.

Winter, R. (2002): Retail Banking im Informationszeitalter - Trends, Geschäftsarchitektur und erste Beispiele, in: Leist, S., Winter, R. (Hrsg.): Retailbanking im Informationszeitalter - Integrierte Gestaltung der Geschäfts-, Prozess- und Applikationsebene, Berlin, S. 29-51. 